\title{
Identification of serum amyloid $A$ in the serum of gastric cancer patients by protein expression profiling
}

\author{
CHIBO LIU ${ }^{1}$, CHUNQIN PAN $^{1}$, JIANMIN SHEN ${ }^{2}$, HAIBAO WANG $^{3}$ and LIANG YONG ${ }^{4}$ \\ Departments of ${ }^{1}$ Clinical Laboratory, and ${ }^{2}$ Radiology; ${ }^{3}$ Hospital Office; ${ }^{4}$ Department of Oncology, \\ Taizhou Municipal Hospital, Taizhou, Zhejiang 318000, P.R. China \\ Received January 16, 2012; Accepted March 28, 2012
}

DOI: $10.3892 / \mathrm{ol} .2012 .664$

\begin{abstract}
The purpose of this study was to screen serum samples from gastric carcinoma patients and to determine whether serum amyloid A protein (SAA) served as a biomarker. SELDI technology was used to screen for changes in SAA levels in the serum samples. A mass cluster with a mass/ charge $(\mathrm{m} / \mathrm{z})$ value between 11.1 and $11.9 \mathrm{kDa}$ was identified in the serum samples from gastric carcinoma patients which was much higher than that of the control group. Furthermore, the increase in this $\mathrm{m} / \mathrm{z}$ peak correlated with the severity of the cancer. High-performance liquid chromatography (HPLC) analysis confirmed that the peak was SAA1. In conclusion, this increase in SAA may be used as a potential biomarker for gastric cancer.
\end{abstract}

\section{Introduction}

Serum proteins often serve as indicators of disease and are a rich source of cancer biomarkers. Certain biomarkers used for the diagnosis, progression and prognosis of cancer have been identified by either surface-enhanced laser desorption/ ionization (SELDI-TOF-MS) (1-7) or matrix-assisted laser desorption/ionization time-of-flight mass spectrometry (MALDI-TOF-MS). SELDI is a useful and powerful platform which may be used to analyze raw mixture samples directly and identify biomarkers in a large number of samples. It provides rapid screening ability and superior visibility of results, avoiding the complication of individual differences (8).

Gastric carcinoma is one of the most common cancer malignancies in Asia. Currently, the most effective treatment for gastric carcinoma is surgical resection; however, prognosis often remains poor due to the advanced stage of cancer progression at diagnosis. Therefore, the identification of novel

Correspondence to: Dr Chibo Liu, Department of Clinical Laboratory, Taizhou Municipal Hospital, Taizhou, Zhejiang 318000, P.R. China

E-mail: liuchibo@126.com

Key words: serum amyloid A, gastric cancer, surface-enhanced laser desorption/ionization time-of-flight mass spectrometry biomarkers for gastric carcinoma, particularly in the early stages, is urgently required.

Potential biomarkers for gastric carcinoma diagnosis were previously reported, including serum amyloid A (SAA) (7), which is also elevated in various types of cancer (9). However, in previous studies, a limited number of samples was used and there was an absence of information on the clinicopathological staging in the patients. In our previous study, sera from a number of gastric carcinoma patients and healthy individuals were screened by ProteinChip array and a classification tree with four biomarkers was established (10). The sensitivity and specificity in blind tests are better than any of the clinical serological tests for gastric carcinoma. However, among the biomarkers discovered by SELDI in various cancers, only a few have been identified, due to technical limitations. In the present study, a new strategy was developed to directly identify the new peptide biomarkers on chip instead of purified biomarkers by MALDI-TOF or other methods.

\section{Materials and methods}

Samples. Samples were obtained from patients in the Taizhou Municipal Hospital, China. The sera of the patients were obtained prior to implementation of any therapeutic measures. The diagnoses were confirmed by postsurgical pathology. The total gastric cancer group consisted of 70 patients at different clinical stages (as defined by the International Union against Cancer, UICC, 1997): stage I ( $n=10)$, stage II $(n=19)$, stage III $(n=11)$ and stage IV $(n=30)$. The median age of the patients was 57 years (range, 32-89). There were 44 males and 28 females. The median ages of patients in the stage I, II, III and IV groups were 53 years (range, 37-77), 64 years (range, 32-77), 58 years (range, 36-71) and 55 years (range, 32-89), respectively. The samples were collected in 10-ml tubes in the early morning before breakfast and then the sera were immediately separated and stored at $-80^{\circ} \mathrm{C}$ until use. The serum samples showed normal clotting time. The study and use of clinical samples was approved by the local ethics committee of medical research, and oral consent was obtained from all subjects.

Analysis of ProteinChip array. After thawing and 2 min of centrifugation $(10,000 \mathrm{rpm}), 5 \mu \mathrm{l}$ serum sample without fractionated treatment was added into $10 \mu 10.5 \% \mathrm{U} 9$ (9 mol/1 urea, $0.2 \%$ CHAPS (3[(3-cholamidopropyl) dimethylammonio]-1- 
propanesulfonate), $0.1 \%$ DTT (DL-dithiothreitol) in a 96-well plate and incubated for $30 \mathrm{~min}$ at $4^{\circ} \mathrm{C}$ with $600 \mathrm{rpm}$ vigorous agitation. The ProteinChip array cassette was placed into a 96-well bioprocessor, and $200 \mu \mathrm{l} \mathrm{NaAc}(50 \mathrm{mmol} / \mathrm{l}, \mathrm{pH} 4.0)$ was added into each well and incubated for $5 \mathrm{~min}$ at $4^{\circ} \mathrm{C}$ with $600 \mathrm{rpm}$ vigorous agitation. The liquid was removed and the procedure was repeated once. NaAC $(185 \mu \mathrm{l})$ was added into each well in the 96 -well plate $(600 \mathrm{rpm}, 2 \mathrm{~min})$ and $100 \mu \mathrm{l}$ samples from the different patient groups were added separately into different wells of the ProteinChip array cassette (600 rpm, $1 \mathrm{~h}$ ). After the content from each well was removed, the wells were washed with $200 \mu 1 \mathrm{NaAC}$ (pH 4.0, $600 \mathrm{rpm}$, $5 \mathrm{~min}$ ). The procedure was repeated two more times. Each spot was washed with $200 \mu$ l high-performance liquid chromatography (HPLC), which was removed immediately. The procedure was repeated once. After air drying, $5 \mu$ l elute was diluted with $5 \mu$ l SPA (saturated solution of sinapinic acid in $50 \%$ acetonitrile with $0.5 \%$ trifluoroacetic acid).

The resulting mixture $(2 \mu 1)$ was aspirated and spotted onto the gold-coated ProteinChip arrays. After air-drying for approximately $5 \mathrm{~min}$ at room temperature, the chips were placed in the protein biological system II-C (PBS II-C) ProteinChip Reader (Ciphergen Biosystems, Inc., Fremont, CA, USA), and data were collected by an average of 150 laser shots, with a detector sensitivity of 8 and a laser intensity of 190. Mass calibration was performed using an all-in-one peptide reference standard comprising vasopressin (1,084.2 Da), somatostatin (1,637.9 Da), bovine insulin $\beta$ chain $(3,495.9 \mathrm{Da})$, human insulin recombinant $(5,807.6 \mathrm{Da})$ and hirudin (7,033.6 Da) (Ciphergen Biosystems, Inc.). The default background subtraction was applied, and the peak intensities were normalized using the total ion current from a mass charge of 1,000-50,000 Da. A biomarker detection software package (Ciphergen Biomarker Wizards, Ciphergen Biosystems, Inc.) was used to autodetect protein peaks. Protein peaks were selected based on a first pass of a signal to noise ratio of 3 and a minimum peak threshold of $20 \%$ of all spectra. This process was completed with a second pass of peak selection at $0.2 \%$ of the mass window, and the estimated peaks were added. These selected protein peaks were averaged as clusters and were exported to a commercially available software package (Biomarker Patterns, Ciphergen Biosystems, Inc.) for further classification analysis.

The profiling spectra of serum samples were normalized using total ion current normalization by Ciphergen's ProteinChip software (version 3.1). Peak labeling was performed using Biomarker Wizard software 3.1 (Ciphergen Biosystems, Inc.). A two-sample t-test was used to compare mean normalized intensities between the case and control groups. $\mathrm{P}<0.05$ was considered statistically significant.

High-performance liquid chromatography (HPLC) analysis. The HPLC separation system consisted of a Shimadzu LC-10ATVP infusion pump, a Shimadzu DGU-14A degasser, a Shimadzu SPD-10A ultraviolet detector, a Rheodyne 7725i admission valve, a Phenomenex Nucleosil C18 chromatographic column (250x4.6 mm ID) and a WDL-95 chromatographic workstation. Linear and gradient elution was carried out with $5 \%$ acetonitrile water solution containing $0.1 \%$ trifluoroacetic acid to $60 \%$ acetonitrile water solution containing $0.1 \%$ trifluoroacetic acid at a rate of $1.0 \mathrm{ml} / \mathrm{min}$ within $0-30 \mathrm{~min}$. Separation and detection were carried out under chromatographic conditions that were measured at UV $220 \mathrm{~nm}$. The effluent of the chromatographic peak was collected in centrifuge tubes for subsequent mass spectrometry analysis.

Matrix-assisted laser desorption/ionization time-of-flight mass spectrometry. MALDI-TOF mass spectra were obtained with a MALDI-TOF mass spectrometer with delayed extraction [Voyager Elite; Applied Biosystems, Inc. (ABI), Framingham, MA, USA]. UV light from a nitrogen laser with a 337-nm emission wavelength was used for irradiation. A typical spectrum was collected at an extraction voltage of $20 \mathrm{kV}$. Delayed (175 ns) extraction mode was used for all acquisitions. The laser power was adjusted to a level just above the threshold for signal appearance to minimize head group loss. Each phospholipid mass spectrum was collected by averaging data from 250 laser shots. For sample plate spotting, $3 \mu \mathrm{l}$ IMACeluted phospholipids were mixed with $3 \mu \mathrm{l}$ matrix, deposited onto the MALDI plate, and allowed to air-dry. The MALDI matrix used in the studies was a recently developed solid ionic crystal matrix consisting of $20 \mathrm{mg}$ paranitroaniline (PNA) and butyric acid at a 1:2 molar ratio dissolved in ethanol.

The spectra were collected using a two-point calibration of protonated lyso-PC at a mass/charge ratio (m/z) of 496.34 and protonated DMPC at $\mathrm{m} / \mathrm{z}$ 678.51. Phospholipids in the biological samples were assigned according to their molecular weights, as derived from either the monoisotopic protonated molecules, monoisotopic sodium adducts, and/or postsource decay (PSD) productions in MALDI-TOF mass spectra.

Database search. Proteins with theoretical matching enzymatic peptides were searched against the ExPASy database (http://us.expasy.org/tools/tagident.html) software according to the $\mathrm{m} / \mathrm{z}$ data of the peptides and proper setting of search parameters. The protein of interest was determined according to the search results and the relative molecular weight of the protein.

\section{Results}

Protein peaks with molecular weights of 11.1-11.9 kDa were significantly increased in the patients with gastric carcinoma. The CM10 ProteinChip was used to carry out the measurements on the serum samples from 70 gastric carcinoma patients and 30 healthy adults. The protein spectra were normalized according to the total ionic strength. Biomarker Wizard software was used to analyze the protein spectra in addition to automatic peak marking, and the signal to noise ratio was set to 3 . The result showed that the average strength of a group of protein peaks with molecular weights of 11.1-11.9 kDa in the gastric carcinoma patients was $18.21 \pm 21.05$ (mean \pm SD), which was significantly higher than that of the normal control at $0.65 \pm 0.55$ (mean $\pm \mathrm{SD})(\mathrm{P}<0.01$; Fig. 1). Corresponding analyses were carried out according to the expression level (peak intensity) of the group of protein peaks with molecular weights of 11.1-11.9 kDa in combination with the clinicopathological staging of the patients. This group of protein peaks was positively correlated with the course of gastric carcinoma (Figs. 1 and 2), and the peak intensity gradually increased with the aggravation of the patient's condition. 


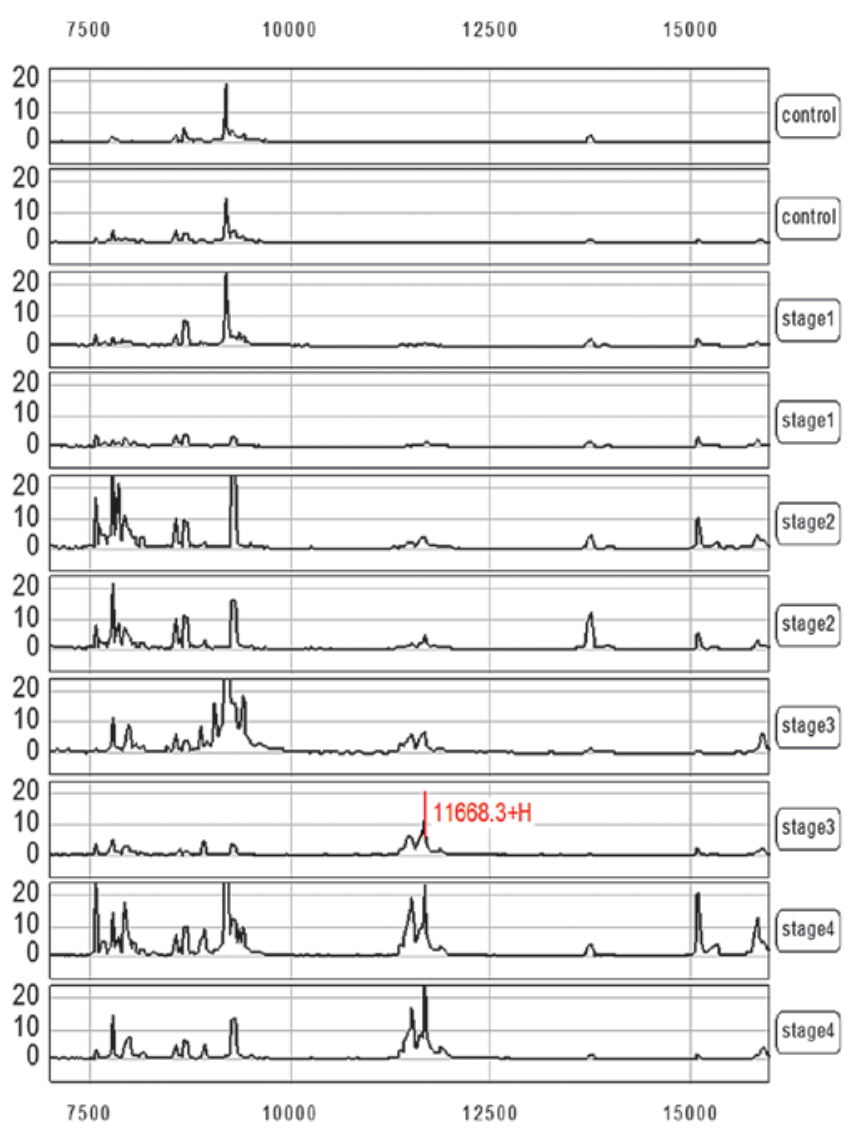

Figure 1. An increased mass cluster in the $\mathrm{m} / \mathrm{z}$ 11.1-11.9 $\mathrm{kDa}$ range in the clinical stages of gastric carcinoma. The spectra of 8 gastric carcinoma patients at stages I-IV are shown from top to bottom. Two patients in each stage were selected.

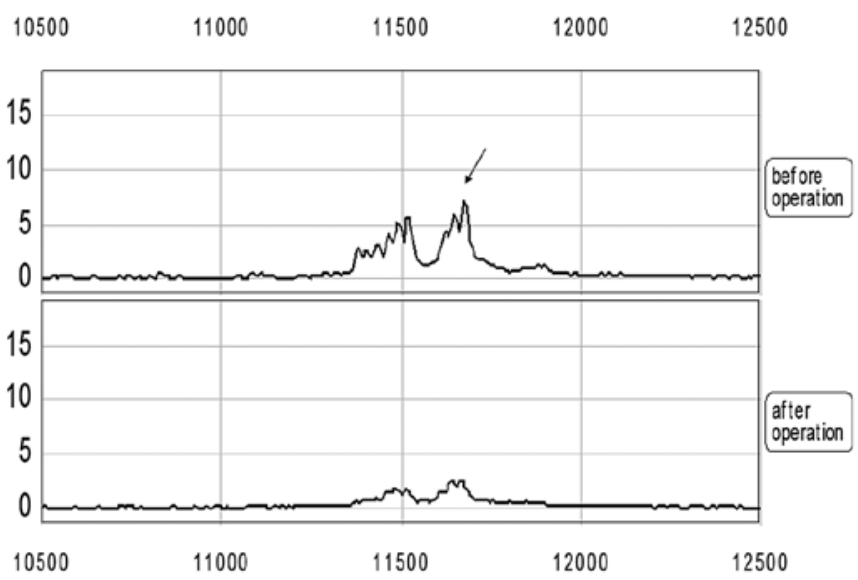

Figure 2. A decreased mass cluster in the $\mathrm{m} / \mathrm{z} 11.1-11.9 \mathrm{kD}$ range in gastric carcinoma patients following surgery. The top two spectra were from one gastric carcinoma patient one week before and one week after surgery.

Protein peaks with molecular weights of 11.1-11.9 kDa on the chip were identified as SAA1. The serum of gastric carcinoma was separated and detected using HPLC, and the effluent of the chromatographic peak was collected in centrifuge tubes. Approximately $1 \mu 1$ solution was dropped on the sample target after the effluent of the chromatographic

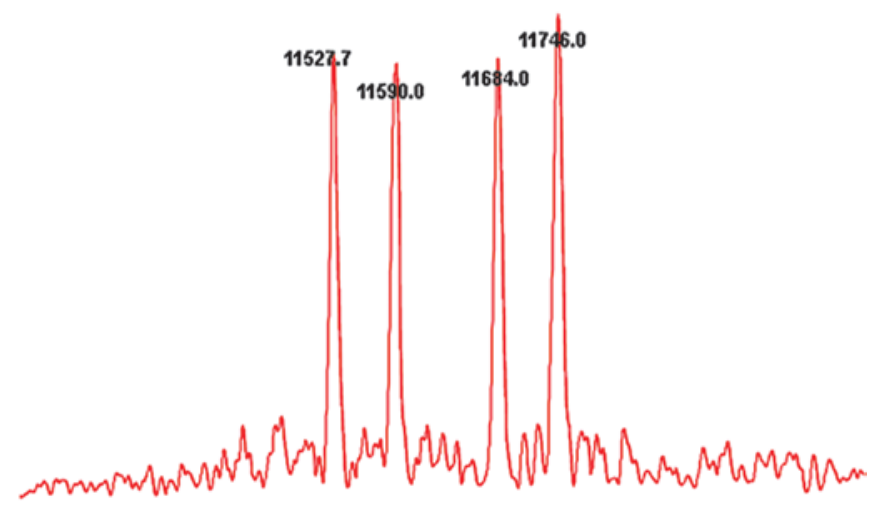

Figure 3. The representative MALDI-TOF mass spectra of gastric carcinoma serum samples.

peak was mixed with the matrix solution. The solution was introduced into the laser desorption/ionization time-of-flight mass spectrometer for analysis once the solvent had evaporated and the sample had crystallized. Signals from 10-50 individual scans were added together to obtain the mass spectra (Fig. 3). Proteins with theoretical matching enzymatic peptides were searched against the ExPASy database (http://us.expasy.org/tools/tagident.html) software according to the $\mathrm{m} / \mathrm{z}$ data of the peptides and proper setting of search parameters, and the group of protein peaks were found to belong to SAA1.

\section{Discussion}

The identification of new biomarkers of human cancers has become more successful due to the development of new high-throughput techniques in the field of proteomics. SELDI-TOF-MS is a promising novel proteomic tool for the detection of biomarkers. It demonstrates superior characteristics including reliable high-throughput ability, speed, few sample requirements, and the ability to analyze complex biological mixtures directly. The efficacy of SELDI in the identification of biomarkers has been proven in cancers of the lung (11), pancreas (12), breast (13), prostate (14) and ovary (15), as well as in other diseases $(16,17)$. However, the disadvantage of SELDI is that it cannot directly identify the biomarkers on chips; therefore it is not widely used in the study of molecular mechanisms. In the present study, one biomarker that may have potential value for diagnosing and monitoring the progression of gastric carcinoma was identified using a new strategy, and its efficacy was found to be consistent with that observed in the study by Cho et al (18). This technique may be applicable in a number of circumstances and facilitate on-chip identification.

SAA is an acute-phase reactant, whose level in blood is elevated in response to trauma, infection, inflammation and neoplasia (18-20). However, SAA is considered to be an acutephase protein existing as various isoforms at a molecular mass of 11.1-11.9 kDa. SAA was found to be significantly higher in patients with metastatic disease than in those with limited disease (21), and may therefore serve as a useful biomarker to monitor relapse, metastasis and prognosis in certain types of cancer (12). Recently, the correlation between SAA and gastric carcinoma was reported by Chan et al (7). Results 
of that study revealed that the SAA level in patients with gastric carcinoma was higher than the SAA level in patients with adenocarcinoma and other diseases, although it is not correlated with tumor size or clinical stage. However, our results suggest that SAA is a more significant biomarker of disease and prognosis in certain types of cancer since it is one of the few biomarkers that corresponds significantly with the progression of gastric carcinoma and may be effectively decreased following medical treatments.

Results of this study showed the average strength of a group of protein peaks with molecular weights of 11.1-11.9 $\mathrm{kDa}$ in the patients of gastric carcinoma to be $18.21 \pm 21.05$ (mean \pm SD), which was significantly higher than the strength of the normal control, at $0.65 \pm 0.55$ (mean $\pm \mathrm{SD})(\mathrm{P}<0.01)$. Corresponding analyses were carried out according to the expression level (peak intensity) of the group of protein peaks with molecular weights of 11.1-11.9 kDa in combination with the clinicopathological staging of the patients. This group of protein peaks was found to be positively correlated with the course of gastric carcinoma. Moreover, the peak intensity gradually increased with the aggravation of the patient's condition.

In conclusion, based on the studies of other authors and our previous study, we suggest that SAA may serve as an effective serum biomarker for the detection of gastric carcinoma and its recurrence.

\section{Acknowledgements}

This study was funded by Zhejiang Medicine, Health and Science grant 2010KYB127, and Zhejiang Gongyixing Applied Technology grant 2011C33045. The authors also thank the Department of Laboratory Medicine, Taizhou Municipal Hospital for its support.

\section{References}

1. Le L, Chi K, Tyldesley S, et al: Identification of serum amyloid A as a biomarker to distinguish prostate cancer patients with bone lesions. Clin Chem 51: 695-707, 2005.

2. Yu Y, Chen S, Wang LS, et al: Prediction of pancreatic cancer by serum biomarkers using surface-enhanced laser desorption/ ionization-based decision tree classification. Oncology 68: 79-86, 2005.

3. Traub F, Feist H, Kreipe HH and Pich A: SELDI-MS-based expression profiling of ductal invasive and lobular invasive human breast carcinomas. Pathol Res Pract 201: 763-770, 2005.

4. Cho WC and Ngan RK: Biomarker discovery in nasopharyngeal carcinoma using proteinchip profiling. J Hong Kong Coll Radiol 11: 63-68, 2008.
5. Cho WC: Proteomics technologies and challenges. Genomics Proteomics Bioinformatics 5: 77-85, 2007.

6. Sasazuki S, Inoue M, Sawada N, et al: Plasma levels of C-reactive protein and serum amyloid A and gastric cancer in a nested casecontrol study: Japan Public Health Center-based prospective study. Carcinogenesis 31: 712-718, 2010.

7. Chan DC, Chen CJ, Chu HC, et al: Evaluation of serum amyloid A as a biomarker for gastric cancer. Ann Surg Oncol 14: 84-93, 2007.

8. Cho WC: Research progress in SELDI-TOF-MS and its clinical applications. Sheng Wu Gong Cheng Xue Bao 22: 871-876, 2006.

9. Woong-Shick A, Sung-Pil P, Su-Mi B, et al: Identification of hemoglobin-alpha and -beta subunits as potential serum biomarkers for the diagnosis and prognosis of ovarian cancer. Cancer Sci 96: 197-201, 2005.

10. Liang Y, Fang M, Li J, et al: Serum proteomic patterns for gastric lesions as revealed by SELDI mass spectrometry. Exp Mol Pathol 81: 176-180, 2006.

11. Valle RP, Chavany C, Zhukov TA and Jendoubi M: New approaches for biomarker discovery in lung cancer. Expert Rev Mol Diagn 3: 55-67, 2003.

12. Yokoi K, Shih LC, Kobayashi R, et al: Serum amyloid A as a tumor marker in sera of nude mice with orthotopic human pancreatic cancer and in plasma of patients with pancreatic cancer. Int J Oncol 27: 1361-1369, 2005.

13. Laronga C, Becker S, Watson P, et al: SELDI-TOF serum profiling for prognostic and diagnostic classification of breast cancers. Dis Markers 19: 229-238, 2003.

14. Adam BL, Qu Y, Davis JW, et al: Serum protein fingerprinting coupled with a pattern-matching algorithm distinguishes prostate cancer from benign prostate hyperplasia and healthy men. Cancer Res 62: 3609-3614, 2002.

15. Petricoin EF, Ardekani AM, Hitt BA, et al: Use of proteomic patterns in serum to identify ovarian cancer. Lancet 359: 572-577, 2002.

16. Cui JF, Liu YK, Zhou HJ, et al: Screening serum hepatocellular carcinoma-associated proteins by SELDI-based protein spectrum analysis. World J Gastroenterol 14: 1257-1262, 2008.

17. Ge Z, Zhu YL, Zhong X, Yu JK and Zheng S: Discovering differential protein expression caused by CagA-induced ERK pathway activation in AGS cells using the SELDI-ProteinChip platform. World J Gastroenterol 14: 554-562, 2008.

18. Cho WC, Yip TT, Ngan RK, et al: ProteinChip array profiling for identification of disease- and chemotherapy-associated biomarkers of nasopharyngeal carcinoma. Clin Chem 53: 241-250, 2007.

19. Malle E and De Beer FC: Human serum amyloid A (SAA) protein: a prominent acute-phase reactant for clinical practice. Eur J Clin Invest 26: 427-435, 1996.

20. Kokubun M, Imafuku Y, Okada M, Ohguchi Y, Ashikawa T, Yamada T and Yoshida H: Serum amyloid A (SAA) concentration varies among rheumatoid arthritis patients estimated by SAA/CRP ratio. Clin Chim Acta 360: 92-102, 2005.

21. Weinstein PS, Skinner M, Sipe JD, Lokich JJ, Zamcheck N and Cohen AS: Acute-phase proteins or tumour markers: the role of SAA, SAP, CRP and CEA as indicators of metastasis in a broad spectrum of neoplastic diseases. Scand J Immunol 19: 193-198, 1984. 